\title{
Prevalence of Multidrug Resistance Mycobacterium Bovis in Human: A Systematic Review and Meta-Analysis
}

\author{
Adel Hussein Elduma ${ }^{1} \quad$ Amin Doosti-Irani $^{3,4}$ Muatsim Ahmed Mohammed Adam ${ }^{5}$ \\ Kourosh HOLAKOUIE-NAIENI ${ }^{*}$ \\ 1.Department of Epidemiology and Biostatistics, School of Public Health, Tehran University of Medical \\ Sciences - International Campus, Tehran, Iran \\ 2.National Public Health Laboratory- Ministry of Health, Sudan \\ 3.Department of Epidemiology, School of Public Health, Hamadan University of Medical Sciences, Hamadan, \\ Iran \\ 4.Research Center for Health Sciences, Hamadan university of Medical Sciences, Hamadan, Iran \\ 5.National Tuberculosis Reference Laboratory, National Public Health Laboratory, Federal Ministry of Health, \\ Khartoum, Sudan \\ 6.Department of Epidemiology and Biostatistics, School of Public Health, Tehran University of Medical \\ Sciences, Tehran, Iran
}

\begin{abstract}
Introduction:

Human infection with multidrug resistant mycobacterium bovis (MDR-M.bovis) is very rare. Recently, infection with this type of bacteria has been described. The WHO strategy to end TB by 2035, recall for more efforts in the diagnosis and treatment of mycobacterium bovis particularly in resource limited countries. The aim of this systematic review was to determine the prevalence of multidrug resistant tuberculosis M.bovis infects human.

\section{Methods:}

International database including Medline, EMBASE, and Web of Sciences, Scopus, and ScienceDirect were searched for related literature. Google Scholar searching engines as well as references list were explored. Studies met the inclusion criteria were included in the systematic review. Random effects model was used to estimate the pooled prevalence.
\end{abstract}

\section{Results:}

A total number of (4275) studies were retrieved and nine studies were included in the meta analysis. The overall estimated prevalence of the multidrug resistant Mycobacterium bovis was 0.04 (95\% CI: 0.02, 0.06). Based one study quality, time and area of study, subgroup analysis were conducted to check for heterogeneity. The prevalence of MDR-M bovis in low quality studies was 0.04 (95\% CI: $0.00,0.06)$, and in high quality studies was 0.05 (95\% CI: 0.02, 0.08).

Conclusions:

The finding of this study indicated that the prevalence of multidrug resistant M.bovis in human is low. However, more attention is needed in the diagnosis and treatment of M.bovis infection in Human.

Keywords: Tuberculosis, Multidrug-Resistant Mycobacterium bovis review meta-analysis Human

DOI: $10.7176 / \mathrm{JHMN} / 69-01$

Publication date: December $31^{\text {st }} 2019$

\section{Introduction}

Tuberculosis is main public health problem in the worldwide, despite the efforts that made to control the disease. According to the World Health Organization report 2016, the estimated new tuberculosis cases were 10.9 million with 1.4 million deaths [1]. Although the infection with Mycobacterium tuberculosis is the main cause of human tuberculosis, there are other species within mycobacterium complex can cause the disease such as M.bovis and $M$. Africanum [2]. M.bovis infects a large range of domesticated and wild animals and infection spread from these animals to humans [3]. Many studies provided evidence of the human-to-human transmission of M.bovis as well as transmission of the pathogen from patients to animals $[4,5]$. M.bovis transmits from infected animals to humans through the consumption of unpasteurized milk and dairy products [6]. But, in recent year due to the currents pasteurization processes infection occur due to close contact through inhalation of droplets or handing of carcasses from infected animals.

Human infection with mycobacterium bovis is very rare and mainly occurs among immune-compromised patients and as nosocomial in HIV patient [5]. Another evidence of human to human transmission of M.bovis has been reported from study conducted in USA [7]. Moreover, M.bovis was accounted for $1.9 \%$ of all reported cases of tuberculosis in Spain between 2004 -2007 [8]. In a separate study conducted in Morocco among 200tuberculosis suspected patients reported $17.8 \%$ infection with m.bovis [9]. In Ethiopia and Nigerian M.bovis infection in human has been reported and accounted for $17.0 \%$ and $15.4 \%$ of all tuberculosis cases respectively $[10,11]$. Moreover, M.bovis has been isolated from sputum samples collected from tuberculosis suspected patients in Egypt and the 
prevalence was $2.2 \%[12]$.

Multi-drug resistance tuberculosis defines as resistance to at least rifampin and isoniazid. In 2015 there were 480000 new multi-drug resistance tuberculosis (MDR-TB) cases according to the World Health Organization report. MDR-TB has become a major health problem not only in developing countries, but Worldwide. MDR-TB cases have been reported in many regions of the World [12]. Although MDR-TB is mainly developed from infection with mycobacterium tuberculosis, MDR-M.bovis outbreaks have been reported [13, 14]. In Spain, M.bovis strains resistant to both rifampin and isoniazid were isolated from tuberculosis patients [8].

Several publications have reported the infection with multidrug resistance due to m.bovis. For instance, one study conducted in Buenos Aires, Argentina indicated that MDR-M.bovis has been reported in 3\% of patients infected with M.bovis[15]. Meanwhile, three samples of MDR-tuberculosis were isolated from 89 human samples of M.bovisin study conducted in Spain was between 2004- 2007 [8]. Furthermore, study conducted in Argentina point out that MDR-M.bovis could be transmitted from human to human where M.bovis was isolated from household contact patients. Two sample of MDR -M.bovis (1\%) were isolated from Mycobacterium tuberculosis complex samples collected from TB patientsin Spain [16]. In a separate study published in 2015 in Mexico, indicated that the rate of the MDR due to M.bovis infection was 38.5\% [17]. These studies showed a significant variability in reporting the MDR tuberculosis that caused by mycobacterium bovis. Thorough investigation is needed to reveal the importance of M.bovis infection in human Worldwide. The aim of this study is to follow systematic review to determine the prevalence of MDR-M.bovis in humans . The aim of this systematic review and meta-analysis was to determine the overall prevalence of the multidrug resistance M.bovis in the world.

\section{Materials and Methods} Searching strategy:

Literature search was conducted independently by two authors (Adel + Mutasim) using the Preferring Reporting Items for Systematic Review and Meta-Analysis (PRISMA) guidelines [18]. Databases searched for literature review included Medline, EMBASE, and Web of Sciences, Scopus, and ScienceDirect. Google Scholar searching engine as well as references list was checked to strengthen the search for missed literatures. Keywords such as MDR-M.bovis, Mycobacterium Bovis, multi-drug resistance and resistant M.bovis were used to extract studies. We used the following strategy to search on Pubmed; \#1 Tuberculosis, Multidrug-Resistant [Mesh terms], \#2MultiDrug Resistant Tuberculosis [tw], \#3 Tuberculosis, MDR [tw], \#4 MDR Tuberculosis [tw].\#5 (\#1 OR \#2 OR \#3 OR \#4).\#6 Prevalence [Mesh terms], \#7 Incidence [Mesh terms], \#8 (\#6 OR \#7), \#9 Mycobacterium bovis [Mesh terms], and \#10 (\#5 AND \#8 AND \#9). Related conference abstract books were searched for unpublished studies.

\section{Studies inclusion criteria:}

Studies that reported Mycobacterium bovis as a causative agent for multidrug resistance tuberculosis in human was retrieved. Studies were searched regardless of their place, publication year and language. Multidrug resistance Mycobacterium bovis studies that reported resist to both Isoniazid and Rifampicin were included in the analysis. The outcome of the interest was the prevalence of multidrug resistance Mycobacterium bovis. Conventional drug susceptibility testing as well as molecular techniques to detect the multidrug resistance were considered as the main test to select studies. Two authors were independently screen the title and abstract of the retrieved studies. If anystudy met the inclusion criteria they reviewed the full contents. The second stage was reviewing the contents of the studies that met the criteria in the first stages. If two authors agreed on study, it would be reviewed in detail. In the case of any disagreement in the selection of studies, it resolved by discussion and judgment by a third author.

\section{Data collection and validity assessment:}

The New Castle-Ottawa Scale (NOS) for assessing the nonrandomized studies in meta-analysis was followed to assess the risk of bias for the extracted studies [19]. Two authors (A and M) were responsible to conduct the quality assessment. Study selection items included (a) criteria to have representative participants; (b) how to reach justified and satisfactory sample size (c) how demographic characteristics of study participants were established; (d) description of the location, setting, date, and related items; (e) methods of the assessment of the outcomes; and (f) Analysis the report of the interested outcomes. Studies were categorized as likely if it satisfied all the criteria, intermediate if it did not meet two criteria, and unlikely if it did not meet more than two criteria. The extracted data included the author, location, year of publication, the sample size, and the diagnostic test. Interested outcome of this study was the number of the multidrug resistant Mycobacterium bovis..

\section{Heterogeneity:}

The heterogeneity among studies was assessed by computing the value of the chi-square $(\mathrm{Q})$ at $10 \%$ significance level. Heterogeneity across the results of studies was quantified by I2, while tau-square (Tue2) was used to assess the between study heterogeneity [20]. 


\section{Statistical analysis:}

The primary outcome measure was the MDR-M.bovis prevalence. The standard error of the prevalence was calculated using binomial probability distribution. Overall and subgroup pooled prevalence was estimated using a random effects model with the inverse variance method. Stata 11 (Stata Corp., College Station, TX, USA) was used for the data analysis. Graphical display of results of each individual study on common scale ( Forest plot) was used to visualize effects of different studies.

\section{Results:}

Multidrug resistant Mycobacterium bovis was reported in Europe in Spain, France, Scotland, Netherland, and Italy [21-25]. In North America, the resistant bacteria of M.bovis had been isolated from patients in USA, Canada and Mexico [26], [27], [28]. Multidrug resistant Mycobacterium bovis has been reported from Argentina [29] in South America, from Burkina Faso in Africa [30], and Pakistan in Asia [31].

A total number of (4275) studies with 16091 participants were retrieved up to December 2017 through searching international database. From retrieved studies, (4112) studies were excluded because they did not relate to the objective of the systematic review. In addition, (128) studies were excluded because of duplication or full text was not accessible. Therefore, 35 full text studies were eligible for in-depth analysis (Figure 1). Then, (20) studies were excluded because they did not meet the eligibility criteria to be included in the Meta analysis. Another six studies were excluded due to small sample size. The remaining studies (9) were met the inclusion criteria for the Meta analysis (Table 1), [32], [33], [34], [35], [36], [37], [38] ], [39], [ 40].

\section{Heterogeneity:}

High heterogeneity was observed among studies included in the Meta-analysis. Based on the results of the heterogeneity tests, the results of included studies were significantly heterogeneous, $\mathrm{I}^{2}=94.1 \%\left(\mathrm{P}=0.001\right.$. The $\mathrm{I}^{2}$ among high quality studies was $94.68 \%$, whereas among low quality heterogeneity was $89.85 \%$. Subgroup analysis based on the quality of studies reduced the heterogeneity among low quality studies.

\section{The prevalence of MDR-M.bovis:}

The pooled estimated prevalence of the multidrug resistant Mycobacterium bovis was 0.04 (95\% CI: 0.02, 0.06) (Figure 2). The prevalence of high quality studies was 0.05 (95\% CI: $0.02,0.08)$, and low quality studies was 0.04 (95\% CI: 0.02, 0.06). The prevalence of MDR-M bovis in Spain was 0.04 (95\% CI: 0.02, 0.06).

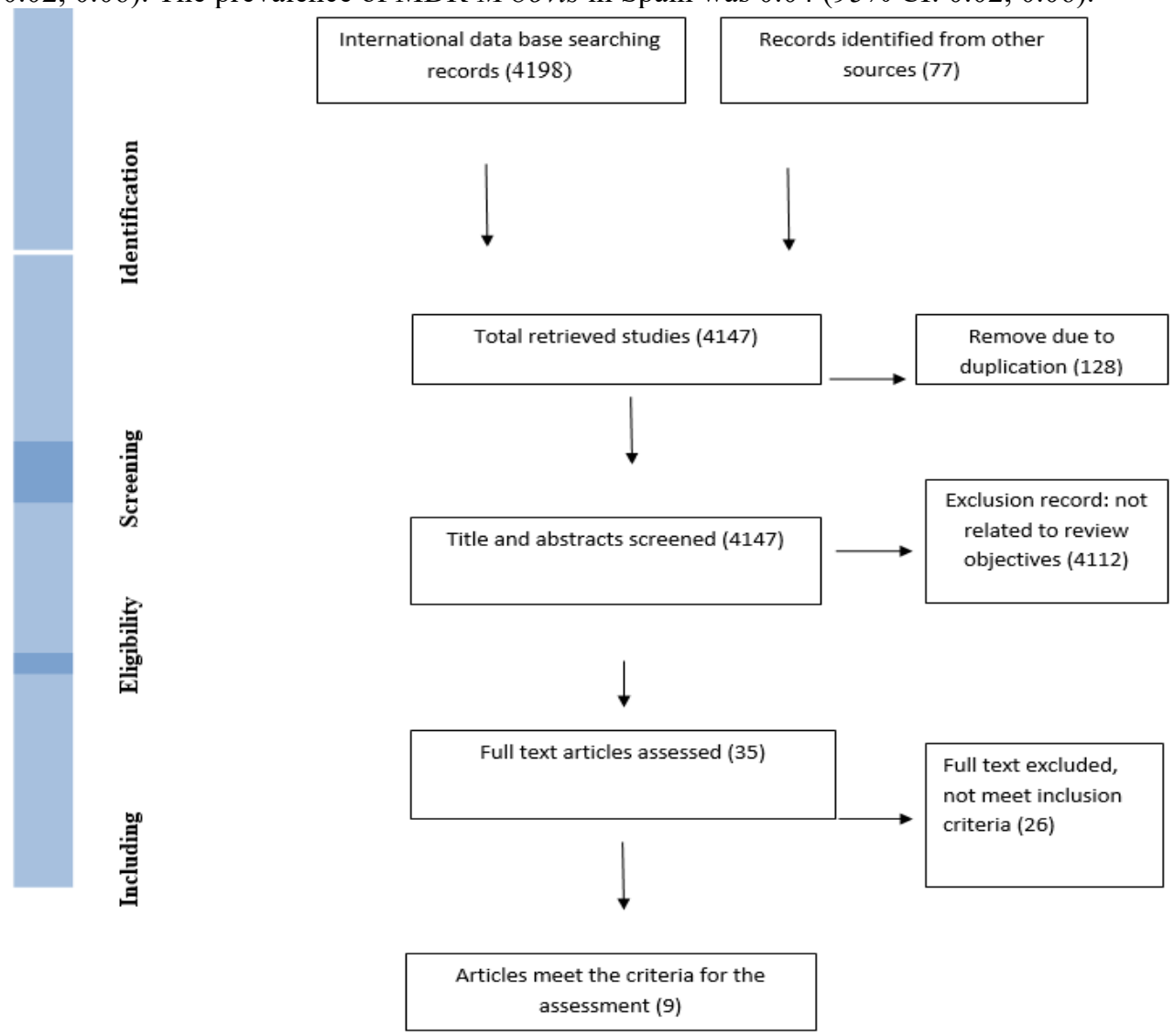

Figure 1: Stages for eligibility criteria and study selection flow chart 
Table 1: Description of studies included in Meta-analysis

\begin{tabular}{|c|c|c|c|c|c|c|c|}
\hline Study & Year & Country & Patients & $\begin{array}{c}\text { Sample } \\
\text { size }\end{array}$ & $\begin{array}{c}\text { Mean } \\
\text { age }\end{array}$ & DST* + Genotyping & $\begin{array}{c}\text { HIV } \\
\text { status }\end{array}$ \\
\hline $\begin{array}{l}\text { Antonio } \\
\text { Guerrero }\end{array}$ & 1997 & Spain & 19 & 434 & 31 & $\begin{array}{c}\text { RFLP**+ } \\
\text { Genotyping }\end{array}$ & HIV \\
\hline Antonio Rivero & 2000 & Spain & 31 & 435 & 35 & DST & HIV \\
\hline J. Cobo & 2001 & Spain & 19 & 1353 & & RFLP & HIV \\
\hline ANNETTE T & 2002 & USA & 2 & 143 & & RFLP + Genotyping. & \\
\hline Carlos Y & 2004 & Spain & 24 & 241 & & DST + RFLP & \\
\hline J. Esteban & 2005 & Spain & 7 & 1366 & 50 & DST & \\
\hline M. Tato & 2006 & Spain & 2 & 55 & & DST + Genotyping. & \\
\hline E. Cordova & 2012 & Argentina & 1 & 97 & & $\begin{array}{ll}\mathrm{PCR}+ & + \\
\mathrm{VNTR}^{*} * * & \text { MIRU- }\end{array}$ & HIV \\
\hline $\begin{array}{l}\text { Miriam } \\
\text { Bobadilla }\end{array}$ & 2015 & Mexico & 89 & 1165 & & DST + MIRU-VNTR & \\
\hline
\end{tabular}

* Drug susceptibility test

** Restriction fragment length polymorphism

*** Variable-number tandem repeats of mycobacterial interspersed repetitive units

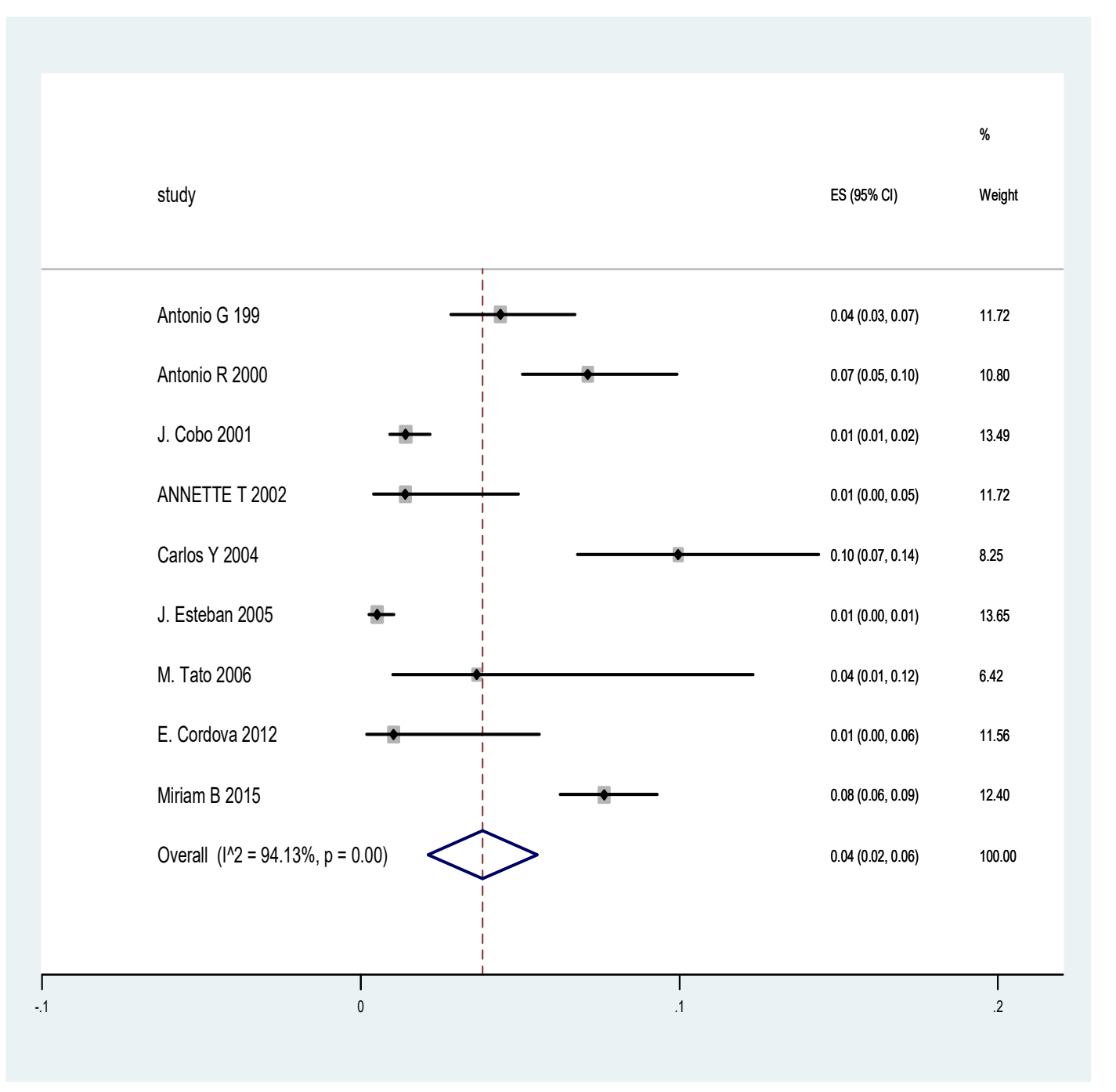

Figure 2: Forest plot of the prevalence of the Multidrug resistant M.bovis

\section{Discussion}

Our study focused on the review of studies reported Mycobacterium bovis resistant to both rifampicin and 
Isoniazid. According to our knowledge, this is a first systematic review and Meta-analysis summarizes articles report the multidrug resistant M.bovis. The Meta analysis findings indicated that the prevalence of multidrug resistant Mycobacterium bovis among human was 1\%. The prevalence of studies conducted before 2010 was $2 \%$, where the prevalence of studies conducted after that was $0.8 \%$. According to theWHO, the global burden of multidrug resistant tuberculosis is 4.1\% [41]. Most of retrieved studies concern with MDR-M bovis were conducted either in Europe or in United State of America (North and South) and only one study was reported from Burkina Faso in Africa. The majority of studies were retrieved from Europe, particularly Spain. It was obvious that developed countries had capacity to investigate different types of mycobacteria causing multidrug resistant. This indicates that the prevalence of MDR Mycobacterium bovis has a considerable decreased.

In spite of the development in molecular technique to diagnose all forms of tuberculosis, this was not reflected in developing countries. Attention should be paid to tuberculosis laboratories in developing countries because they have high burden of tuberculosis infection. It is important to consider the occurrence of MDR M.bovis because many studies provided evidence that this pathogen has been isolated from animals. A recent study published in 2017 reported that Mycobacterium bovis resistant to both rifampicin and isoniazid in a study conducted in Brazil [42].

It is worth mentioning that a considerable number of laboratories in developing countries do not have the capacity to do mycobacterium culture and depend mainly on microscopic examination. In addition, laboratories that have culture for Mycobacterium pathogen, they don't considere M.bovis in their routine work for a reason that this bacterium cannot grow in Media with Glycerol 43]. Also, M.bovis is naturally resisted to pyrazinamide so if the bacteria did not consider in the diagnosis of tuberculosis patient, this may lead to resistant phenomenon due to improper treatment. The WHO Report indicated that new TB case confirmed by bacteriology to test the drug susceptibility was very low ( $12 \%$ out of 2.7 million new cases) [44]. Tuberculosis laboratory that has the capacity to do bacterial culture should add pyruvate inculture media. Many studies correlate the occurrence of multidrug resistant mycobacterium bovis in animals and human in Mexico and USA 45].

. According to the finding of this systematic review and meta-analysis, Bovine tuberculosis is decreasing in Europe and North America where the last report was from Spain in 2009. It was obvious that the prevalence of human Mycobacterium bovis decreases after the implementing of prevention programme in Europe and North America[46]. On the other hand, recent report of multidrug resistant Mycobacterium bovis was from Burkina Faso in 2017. This is the only study reported report the occurrence of this diseases in African continent [30].

Although bovine tuberculosis is prevalent in African countries, measures to control this disease such as pasteurization and test-slaughter strategy are not applied 47]. Other factors might contribute in the risk to get the disease is the human behavior where people have close contact with animals particularly in rural areas. Also, high prevalence of HIV increases the risk of getting the disease [48]. So, this leads to that human more susceptible to infect with bovine tuberculosis. In Africa, 90 countries have reported the occurrence of Mycobacterium bovis among cattle population [49]. More efforts are required to reduce the burden of disease in Human and animals. This could be achieverd by setup a test slaughter strategy putting in mou mind a suitable compensation policy in Africa. Polices, diseases surveillance system and clinical diagnose have to take $M$. bovis in to consideration.

Study conducted in Netherland reported M.bovis which was resisted multiple second line drugs. This means that M.bovis not only resist to multiple first line tuberculosis drugs, but also resist to multiple second line drugs and might lead to Extensively drug resistant mycobacterium (XDR) [50]. WHO end strategy to end TB by 2035 , recall for more efforts in the diagnosis and treatment of Mycobacterium bovis particularly in resource limited countries. The strategy of early diagnosis of tuberculosis and drug susceptibility testing should be considered to identify multidrug resistant mycobacterium bovis [51]. WHO, FAO, and World Organization for Animal Health together published the Roadmap of zoonotic tuberculosis in 2017 in order to highlight the need in defining different groups at risk to get the disease. In addition, funding bodies have to address the detection and treatment of $M$. bovis activities by allocating funding to these activities [52].

This study has many limitations which include that the number studies selected for the Meta analysis was low. In addition, some studies have small sample size which might increase the risk of selection bias. The searching process included only articles that published in English. In addition, the systematic review and meta-analysis was based on published studies available online.

\section{Conclusion}

The systematic review provided evidence that multidrug resistant mycobacterium bovis infect human. According to the meta-analysis, the prevalence of this bacterium is low and more research is needed to figure out the exact burden of MDR-M. bovis particularly in low and middle income countries. Although this study was to estimate the pooled prevalence of multidrug resistant mycobacterium bovis using systematic review and Meta Analysis, it did not reflect the accurate prevalence because of the heterogeneity in the included studies. 


\section{Author Contributions:}

Adel Elduma and Muatsim Adam review and select articles for the systematic review, Adel Elduma write the manuscript, Dr. Amin Doosti-Irani revise the article, Professor Kourosh HOLAKOUIE-NAIENI conceptualization and general supervision.

\section{Acknowledgments}

The authors would like to thank Dr. Hamdan Mostafa Hamdan, MDR-Focal person at the national tuberculosis programme in Sudan for his valuable comments

\section{Conflicts of Interest:}

The authors declare no conflict of interest for this article

\section{References}

1. World Health Organization. Global tuberculosis report 2017. 2017; Available from: http://www.who.int/tb/publications/global_report/en/.

2. Niemann S, R.E., Rusch GS. among members of theMycobacterium tuberculosis complex by molecular and biochemical features: evidence for two pyrazinamide-susceptible subtypes of M. bovis. J Clin Microbiol 2000. 38: p. 15257.

3. Van Soolingen D, D.H.P., Haagsma $\mathrm{J}$ et al, Use of various genetic markers in differentiation of Mycobacterium bovis strains from animals and humans and for studying epidemiology of bovine tuberculosis. J Clin Microbiol., 1994. 32(10): p. 2425-33.

4. Fritsche A1, E.R., Buhl D, Zellweger JP. Mycobacterium bovis tuberculosis: from animal to man and back. Int J Tuberc Lung Dis, 2004. 8(7): p. 903-4.

5. Evans JT1, S.E., Banerjee A, Smith RM, Dale J, Innes JA, Hunt D, Tweddell A, Wood A, Anderson C, Hewinson RG, Smith NH, Hawkey PM, Sonnenberg P. Cluster of human tuberculosis caused by Mycobacterium bovis: evidence for person-to-person transmission in the UK. Lancet, 2007. 369(9569).

6. Thoen C1, L.P., de Kantor I. The importance of Mycobacterium bovis as a zoonosis. Vet Microbiol., 2006. 112(2-4): p. 339-45.

7. Moonan, C.S. et.al. Human Tuberculosis Caused by Mycobacterium bovis in the United States, 2006-2013. Clin Infect Dis., 2016. 63(5): p. 594-601.

8. E. Rodríguez, L.P.S., S. Pérez, L. Herrera, M. S. Jiménez, S. Samper, M. J. Iglesias. Human tuberculosis due to Mycobacterium bovis and M. caprae in Spain, 2004-2007. INT J TUBERC LUNG DIS 2009. 13(12).

9. Mahamat Fayiz Abakar, H.Y.A., Philipp Justus Bless, Lisa Crump, Petra Lohmann, Mirjam Laager, Nakul Chitnis, Jakob Zinsstag. Transmission dynamics and elimination potential of zoonotic tuberculosis in morocco. PLoS Neglected Tropical Diseases 2017.

10. J.E. Shitaye, W.T., I. Pavlik. Bovine tuberculosis infection in animal and human populations in Ethiopia: A review. Veterinarni Medicina, 2007. 52(8): p. 317-332.

11. JD Mawak, c.a.N.G., CSS Bello, and YT Kandakai-Olukemi. Human Pulmonary Infections with Bovine and Environment (Atypical) Mycobacteria in Jos, Nigeria. Ghana Med J, 2006. 40(4): p. 132-136.

12. World Health Organization. Treatment of Tuberculosis: Guidelines for National Programmes. Third Edition. 2003; Available from: http://apps.who.int/iris/bitstream/10665/67890/1/WHO_CDS_TB 2003.313 eng.pdf.

13. Martín, A.R , et.al. High Rate of Tuberculosis Reinfection during a Nosocomial Outbreak of MultidrugResistant Tuberculosis Caused by Mycobacterium bovis Strain B. Clinical Infectious Diseases, 2001. 32(1): p. 159-161.

14. Blázquez J, E.d.L.M.L., Samper S, Martín C, Guerrero A, Cobo J, Van Embden J, Baquero F, GómezMampaso E. Genetic characterization of multidrug-resistant Mycobacterium bovis strains from a hospital outbreak involving human immunodeficiency virus-positive patients. J Clin Microbiol. , 1997. 35(6): p. 13903.

15. Cordova E1, G.X., Boschi A, Lossa M, Robles M, Poggi S, Ambroggi M. Human Mycobacterium bovis infection in Buenos Aires: epidemiology, microbiology and clinical presentation. Int $\mathrm{J}$ Tuberc Lung Dis. , 2012. 16(3): p. 415-7.

16. Samper S, I.M., Rabanaque MJ, Gómez LI, Lafoz MC, Jiménez MS, Ortega A, Lezcano MA, Van Soolingen D, Martín C. Systematic molecular characterization of multidrug-resistant Mycobacterium tuberculosis complex isolates from Spain. J Clin Microbiol. , 2005. 43(3): p. 1220-7.

17. Miriam Bobadilla-del Valle, e.a. Trends of Mycobacterium bovis Isolation and First-Line Anti-tuberculosis Drug Susceptibility Profile: A Fifteen-Year Laboratory-Based Surveillance. PLoS Negl Trop Dis 2015. 9(9).

18. Moher D, L.A., Tetzlaff J, Altman DG; PRISMA Group. Preferred reporting items for systematic reviews and meta-analyses: the PRISMA statement. Ann Intern Med., 2009. 151(4): p. 264-9.

19. Wells G, S.B., O'connell D, et al. The Newcastle-Ottawa Scale (NOS) for assessing the quality of 
nonrandomised studies in meta-analyses. 2000; from: http://www.ohri.ca/programs/clinical_epidemiology/oxford.asp.

20. Higgins JP, Thompson GS, Deeks JJ, Altman D.Measuring Inconsistancy in meta-analysis. BMJ. 2003; 327 (7414): $557-60$

21. Samper S, M.C., Pinedo A, Rivero A, Blázquez J, Baquero F, van Soolingen D, van Embden J. Transmission between HIV-infected patients of multidrug-resistant tuberculosis caused by Mycobacterium bovis AIDS, 1997. 11: p. 1237-1242.

22. Bouvet E, Mendoza-Sassi G, Lariven S, Vallée E, Pernet M, Gottot S, Vachon F. A nosocomial outbreak of multidrug-resistant Mycobacterium bovis among HIV-infected patients. A case-control study. AIDS, 1993. 7: p. $1453-1460$.

23. Hughes VM, S.R., Doig C, Stevenson K, Sharp JM, et al. Analysis of multidrug-resistant Mycobacterium bovis from three clinical samples from Scotland. Int J Tuberc Lung Dis 2003. 7: p. 1191-1198.

24. Schultsz C, K.E., van Soolingen D, Prins JM. Disseminated infection due to multidrug-resistant Mycobacterium bovis in a patient who was seropositive for human immunodeficiency virus. Clin Infect Dis., 1996. 23(4): p. 841-3.

25. Sechi LA, Z.S., Sanguinetti M, et al. Molecular Basis of Rifampin and Isoniazid Resistance in Mycobacterium bovis Strains Isolated in Sardinia, Italy. Antimicrobial Agents and Chemotherapy, 2001. 45(6): p. 1645-1648.

26. Nitta AT, K.L., Kim J, et al. Limited transmission of multidrug-resistant tuberculosis despite a high proportion of infectious cases in Los Angeles County, California. Am J Respir Crit Care Med, 2002. 165: p. 812-17.

27. Long R, N.E., Chomyc S, van Embden JAN, McNamee C, et al. Transcontinental Spread of Multidrugresistant Mycobacterium bovis. American Journal of Respiratory and Critical Care Medicine 1999. 159: p. 2014-2017.

28. Laniado-Laborin R, M.-S.R., et.al. Molecular characterization of Mycobacterium bovis isolates from patients with tuberculosis in Baja California, Mexico. Infect Genet Evol, 2014. 27: p. 1-5.

29. Etchechoury I, V.G., Morcillo N, et al. Molecular typing of Mycobacterium bovis isolates in Argentina: first description of a person-to-person transmission case. Zoonoses Public Health, 2010. 57: p. 375-81.

30. Diagbouga S, N.C., Tarnagda Z, Djibougou A, Henry N, et al. Mycobacterium bovis Prevalence in Humans Does Not Differ Between Regions in Burkina Faso. Arch Clin Microbiol, 2017. 8: p. 1-6.

31. Munir S, M.N., Shahid S, Khan MI. Molecular detection of Isoniazid, Rifampin and Ethambutol resistance to M. tuberculosis and M. bovis in multidrug resistant tuberculosis (MDR-TB) patients in Pakistan. Microb Pathog, 2017. 110: p. 262-274.

32. Antonio Guerrero, J.C., et.al. Nosocomial transmission of Mycobacterium bovis resistant to 11 drugs in people with advanced HIV-1 infection. Lancet 1997. 350: p. 1738-42.

33. Antonio Rivero, M.M.r., et.al. High Rate of Tuberculosis Reinfection during a Nosocomial Outbreak of Multidrug-Resistant Tuberculosis Caused by Mycobacterium bovis Strain B. Clinical Infectious Diseases, 2001. 32: p. 159-61.

34. J. Cobo, Á.A., Moreno, E. et.al. Risk factors for nosocomial transmission of multidrug-resistant tuberculosis due to Mycobacterium bovis among HIV-infected patients. INT J TUBERC LUNG DIS, 2001. 5(5): p. 413418.

35. ANNETTET. NITTA, e.a. Limited Transmission of Multidrug-Resistant Tuberculosis Despite a High Proportion of Infectious Cases in Los Angeles County, California American Journal of Respiratory and Critical Care Medicine, 2002. 165(6): p. 812-817.

36. Carlos Y. Soto, M.C.M.n., et.al. IS6110 Mediates Increased Transcription of the phoP Virulence Gene in a Multidrug-Resistant Clinical Isolate Responsible for Tuberculosis Outbreaks. JOURNAL OF CLINICAL MICROBIOLOGY,, 2004. 42(1): p. 212-219.

37. S. Samper, M.J.I., et.al. Systematic Molecular Characterization of Multidrug-Resistant Mycobacterium tuberculosis Complex Isolates from Spain. JOURNAL OF CLINICAL MICROBIOLOGY,, 2005. 43(3): p. $1220-1227$

38. Tato M, d.1.P.E., et.al. In vitro activity of linezolid against Mycobacterium tuberculosis complex, including multidrug-resistant Mycobacterium bovis isolates. Int J Antimicrob Agents, 2006. 28(1): p. 75-8.

39. E. Cordova, X.G., A. Boschi, et.al. Human Mycobacterium bovis infection in Buenos Aires: epidemiology, microbiology and clinical presentation. INT J TUBERC LUNG DIS, 2012. 16(3): p. 415-417.

40. Miriam Bobadilla-del Valle, et.al. Trends of Mycobacterium bovis Isolation and First-Line Anti-tuberculosis Drug Susceptibility Profile: A Fifteen-Year Laboratory-Based Surveillance. PLOS Neglected Tropical Diseases, 2015. 9(9): p. e0004124.

41. World Health Organization. Drug-resistant TB: global situation. 2016; Available from: http://www.who.int/tb/areas-of-work/drug-resistant-tb/global-situation/en/.

42. Franco MMJ, R.M., et.al. Genotyping and rifampicin and isoniazid resistance in Mycobacterium bovis strains 
isolated from the lymph nodes of slaughtered cattle Tuberculosis, 2017. 104: p. 30 -37.

43. Grange JM, Y.M., de Kantor IN. Guidelines for speciation within the Mycobacterium tuberculosis complex. WHO, 1996.

44. World Health Organization. WHO Global Tuberculosis Report 2015 [cited 2016 March 1]; Available from: http://www.who.int/tb/publications/global_report/en/

45. Rodwell TC, K.A., Moore M, et al. Tracing the origins of Mycobacterium bovis tuberculosis in humans in the USA to cattle in Mexico using spoligotyping. Int J Infect Dis 2006: p. e129-135.

46. de la Rua- Domenech R. Human Mycobacterium bovis infection in the United Kingdom: Incidence, risks, control measures and review of the zoonotic aspects of bovine tuberculosis. Tuberculosis, 2006. 86(2): p. 77109.

47. Cosivi O, G.J., et al. Zoonotic tuberculosis due to Mycobacterium bovis in developing countries. Emerg Infect Dis, 1998. 4(59-70).

48. Ayele WY, N.S., Zinsstag J, Weiss MG, et,al. Bovine tuberculosis: an old disease but a new threat to Africa. Int J Tuberc Lung Dis, 2004. 8: p. 924 - 37.

49. World Organization for Animal Health (OIE). World Animal Health Information System (WAHIS) interface. 2015 [cited 2015 March 24]; Available from: http://www.oie.int/wahis 2/public/wahid.php/Wahidhome/Home.

50. J. van Ingen, et.al. Second-line drug resistance in multidrug-resistant tuberculosis cases of various origins in the Netherlands. INT J TUBERC LUNG DIS, 2008. 12(11): p. 1295-1299.

51. World HealthOrganization. 2015. Gear up to end TB-introducing the WHO End TB Strategy. 2015 [cited 2015 May 10]; Available from: http://www.who.int/tb/EndTBadvocacy_brochure/en/.

52. World HealthOrganization. Roadmap for zoonotic tuberculosis 2017; Available from: http://www.who.int/tb/publications/2017/zoonotic_TB/en/. 